\title{
Évaluation des caractéristiques des stomates chez le palmier à huile (Elaeis guineensis Jacq.)
}

\author{
Alain Ignassou DJINET ${ }^{1}$, Joseph Martin BELL² ${ }^{2}$ Rasmata NANA ${ }^{3}$, Memti Mberdoum NGUINAMBAYE ${ }^{4}$, \\ Zoumbiéssé TAMINI ${ }^{3}$ \\ 1 École Normale Supérieure de Bongor, Département des Sciences de la Vie et de la Terre BP 15 Bongor, Tchad. \\ 2 Université de Yaoundé I, Faculté des Sciences, Département de Biologie et Physiologie Végétales BP 812 \\ Yaoundé, Cameroun. \\ 3 Université Ouaga I Pr. Joseph KI-ZERBO, Unité de Formation et de Recherche en Sciences de la Vie et de la \\ Terre, Laboratoire de Biosciences Équipe d'Écophysiologie Végétale 03BP7021 Ouagadougou, Burkina Faso \\ ${ }^{4}$ Université de Ndjaména Faculté des Sciences Exactes et Appliquées, Département de Biologie, BP N'djaména, \\ Tchad. \\ Auteur de correspondance : DJINET Ignassou Alain Adresse BP 15 Bongor, e-mail : alain djinet@yahoo.fr, Téléphone \\ $+23566438543$
}

Original submitted in on 12th May 2016. Published online at www.m.elewa.org on 31 $31^{\text {st }}$ August 2016 http://dx.doi.org/10.4314/jab.v104i1.2

\section{RÉSUMÉ}

Objectif : l'objectif principal de cette étude est de déterminer les paramètres caractéristiques des stomates chez Elaeis guineensis Jacq actuel.

Méthodologie et résultats : Les paramètres comme la densité, la longueur et la largeur des stomates ont été évalués en observant l'épiderme des feuilles au microscope. Ainsi, le matériel adulte (dura, pisifera et, tenera) en champ et le matériel jeune (plants de tenera en pépinière et plants de tenera en pré pépinière) ont été considérés. Les résultats ont montré que, sur la face inférieure des feuilles, la densité stomatique est plus importante chez le matériel adulte 50,32 stomates par $\mathrm{mm}^{2}$ que chez le matériel jeune 23,88 stomates par $\mathrm{mm}^{2}$ et, sur la face supérieure des feuilles, cette situation s'inverse 4,77 stomates par $\mathrm{mm}^{2}$ contre 9,21 stomates par $\mathrm{mm}^{2}$. Les stomates sur la face supérieure des feuilles sont plus longs et larges que ceux observés sur la face inférieure des feuilles pour tous les matériels. Les stomates du matériel adulte sont aussi plus longs que ceux du matériel jeune. Pour tous les paramètres étudiés, la face inférieure apparaît la plus indiquée pour caractériser des stomates chez le palmier à huile.

Conclusion et application des résultats : cette présente étude montre l'importance des stomates dans la caractérisation du niveau de ploïdie du palmier à huile. Ainsi, les résultats obtenus peuvent s'appliquer dans les recherches des plantes ayant une fertilité plus intéressante en s'appuyant sur la polyplö̈disation.

Mots clés : Elaeis guineensis, stomates, caractéristiques.

\section{ABSTRACT}

Objective: To determine the characteristics of stomata parameters in Elaeis guineensis Jacq. Current.

Methodology and results: Parameters such as the density, the length and with of stomata were evaluated by observing the leaf epidermis microscopically. Thus, adult material (dura, pisifera and tenera) field and the young material (tenera nursery plant and prenursery tenera plant) were considered. The results showed that on 
the underside of leaves, stomata density was greater in adult material 50.32 stomata per $\mathrm{mm}^{2}$ than in younger material 23.88 stomata per $\mathrm{mm}^{2}$ on the upper leaf surface this reverses stomata per $\mathrm{mm}^{2} 4.77$ against 9.21 stomata per $\mathrm{mm}^{2}$. Stomata on the upper leaf surface are longer and wider than those seen on the underside of leaves for all materials. Stomata adult materials are also longer than those of young material. For all the parameters studied, the underside of leaves appears most indicated to characteristerize the stomata in the oil palm.

Conclusion and application of results: this present study shows the importance of stomata in the characterization of the ploidy level of the oil palm. So, results can be applied in the research of plant with a more interesting fertility based on the polyploidization.

Keywords: Elaeis guineensis, stomata, characteristics

\section{INTRODUCTION}

Le palmier à huile est l'une des principales plantes oléagineuses. II est cultivé pour son fruit, une drupe, exploitée pour sa pulpe qui fournit l'huile de palme et pour son amande qui contient l'huile de palmiste. Mais, la quantité de ces huiles produites semble insuffisante, face à la demande de plus en plus croissante due à la forte croissance démographique (Demol, 2002). La méthode de sélection par croisement s'est montrée limitée car dans la population du palmier à huile supposée hybrides tenera on rencontre encore des individus de moindre intérêt entraînant ainsi une faible production. Cette situation nous impose une recherche des méthodes pouvant nous amener à obtenir des individus plus vigoureux et fixés. L'exploitation des phénomènes de totipotence et de régénération a conduit à l'introduction de nouvelles méthodes telles que l'haplodiploïdie (Dubois, 1989). Elle présente un grand intérêt pour l'amélioration des plantes car elle permet d'accélérer le cycle de sélection (Auge et al.,

\section{MATÉRIEL ET MÉTHODES}

Matériel végétal: Le matériel végétal a été représenté par les feuilles de trois(3) variétés du palmier à huile (dura, tenera et pisifera). Le matériel végétal provient d'un champ, d'une prépepinière et d'une pépinière. Le matériel a été prélevé sur les pieds de dix(10) ans dans les palmeraies environnantes au sein du campus de l'Université de Yaoundé I, tandis que le matériel de prépépinière et pépinière uniquement de la variété tenera provenait de l'IRAD (Institut de Recherche Agronomique pour le Développement) et de CERES (Centre de Recherche, d'Études et d'Exploitation des semences) Sarl.

Méthodes
1989). En effet, les stomates jouent non seulement un rôle physiologique fondamental dans la vie de la plante (photosynthèse, transpiration), mais ils peuvent représenter aussi des éléments taxonomiques d'un certain intérêt (Scienza et Boselit, 1981). Par ailleurs, les caractéristiques des stomates peuvent être utilisées pour l'évaluation précoce de la résistance à la sécheresse ou pour déterminer la vigueur de la plante. En outre, les stomates peuvent être aussi utilisés comme des indicateurs de la pollution locale de l'air (Koffi et al., 2014). L'objectif principal de ce travail est de déterminer les paramètres caractéristiques des stomates chez le palmier à huile normal diploïde afin d'appliquer dans le futur les méthodes de la Biotechnologie. Les objectifs spécifiques portent sur l'évaluation de la densité, de la longueur et de la largeur des stomates des feuilles de palmier à huile en fonction des variétés, de l'âge de la plante et des faces des feuilles.

Détermination variétale: La méthode de détermination variétale utilisée est l'examen de la section du fruit des différents plants adultes des variétés dura, tenera et pisifera.

Prélèvement des échantillons: Pour ce travail, les échantillons ont été prélevés à midi et préparés en vue d'une observation au microscope. De ce fait trois variétés de palmier à huile (dura, tenera et pisifera) en champ d'âge environ dix ans et le plant de tenera en prépépinière $(4 \mathrm{mois})$ et pépinière $(12 \mathrm{mois})$ notés respectivement tenera $a_{p p}$ et tenera $a_{p}$ ont été considérés. Pour chaque matériel et type d'âge considéré, les échantillons ont été prélevés sur trois(3) feuilles notées respectivement $F 1, F 2$ et $F 3$ à des niveaux différents. 
Pour chaque feuille trois(3) folioles ont été retenues respectivement à la base, au milieu et à la pointe Mounir et Raoult (2000). Les échantillons ont été préparés sur les deux(2) faces de la feuille: la face supérieure ou adaxiale et la face inférieure ou abaxiale. Pour chaque variété quatre(4) répétitions ont été faites, cinq(5) matériels utilisés, soit un total de vingt plantes ciblées pour l'étude.

Préparation des échantillons et observations: L'observation de l'épiderme nécessite l'élimination du parenchyme chlorophyllien dont l'épaisseur empêche l'observation des cellules épidermiques. Pour cela, une lame de rasoir a été placée à la surface de la feuille puis l'épiderme transparent se trouvant à la surface de celle-ci est soulevé doucement. Le fragment de l'épiderme transparent obtenu constitue l'échantillon qui peut être observé. Il est alors recouvert d'une lame et lamelle puis coloré par le lugol pour une observation au microscope. Cette observation a permis d'identifier les stomates et de mettre en évidence leur organisation. Les stomates ainsi identifiés sont comptés, la longueur et la largeur de chacun sont mesurées.

Densité stomatique: La densité stomatique est le nombre des stomates par unité de surface. Pour déterminer cette densité stomatique, l'objectif X40 du microscope muni d'un micromètre oculaire a été utilisé. Un point est fixé puis le micromètre est placé de manière à obtenir un carré. Ensuite les stomates existants sur la face délimitée sont comptés et le calcul de champ d'un millimètre carré de surface a été fait. Pour chaque feuille, 3 mesures ont été faites sur la face inferieure tout comme sur la face supérieure, soit 6 mesures par feuille. 3 feuilles par plante ont été considérées, soit 18 mesures par plante. Pour chaque matériel utilisé, 72 mesures ont été effectuées, 36 sur la face inferieure et 36 sur la face supérieure.

Longueur et largeur des stomates: Les mesures de la longueur et de la largeur ont été faites avec l'objectif à immersion. Pour ce dernier cas, le micromètre est placé dans le sens de la longueur et de la largeur des stomates permettant donc de mesurer ces paramètres. Ainsi, 5 stomates ont été choisis au hasard par mesure. Trois mesures ont été effectuées par feuille, on a 15 stomates choisis par feuille, soit 45 stomates dont la longueur et la largeur ont été mesurées pour une plante. Pour chaque matériel 4 plantes ont été retenues soit 180 stomates mesurés. Pour les 5 matériels (dura, tenera, pisifera, tenera pépinière et tenera prépépinière) 900 stomates ont été mesurés sur la face inferieure des feuilles. Sur la face supérieure des feuilles, compte tenu du nombre limité des stomates, cette méthode n'a pas été appliquée, seulement 224 mesures qui correspondent à 224 stomates ont été faites soit 36 mesures pour la variété dura, 38 pour la variété tenera et 35 pour la variété pisifera, 52 pour le matériel tenera pépinière et 63 pour le matériel tenera prépépinière.

Méthode statistique: Les résultats relatifs aux paramètres évalués ont été soumis à une analyse de variance(ANOVA). Les tests de comparaison des moyennes ont été effectués selon la méthode de Fisher au seuil de $5 \%$. Le logiciel utilisé pour traiter les données est XLSTAT version 7.5.2

\section{RÉSULTATS}

La figure $1(A, B$ et $C)$ présente les stomates des trois(3) variétés de palmier à huile.

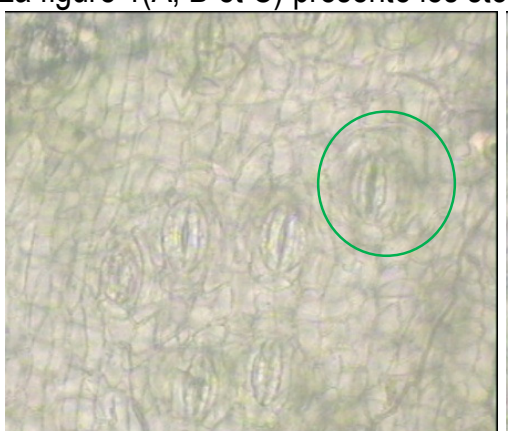

A : Dura

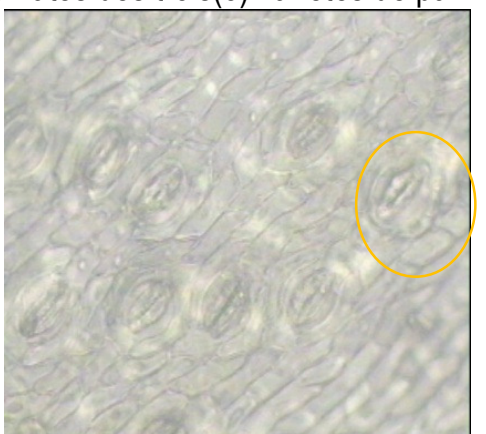

B : Pisifera

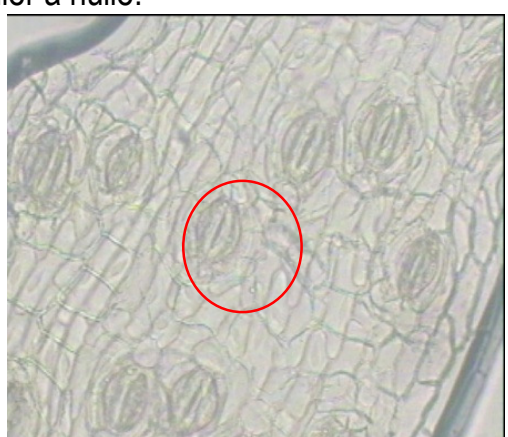

C: Tenera

Figure 1 : Stomates à la face inférieure des feuilles des différentes variétés de palmier à huile $a-$ variété dura ; $b$ variété pisifera ; c- variété tenera.

Les résultats des analyses statistiques sont présentés dans les tableaux1 et 2. Les résultats montrent que la densité stomatique varie en fonction des variétés, de l'âge des plants et les faces des feuilles. II existe une différence significative au seuil de $5 \%$ avec $\mathrm{P}<0,0001$ entre les matériels pour la face supérieure et la face 
inferieure des feuilles. Sur la face supérieure les densités des stomates ont varié de 4,11 à 9,21 stomates par $\mathrm{mm}^{2}$. La plus grande densité est notée chez le matériel Tenera pépinière et la plus faible densité est enregistrée chez la variété Dura. Cependant, sur la face inférieure des feuilles, la valeur de la densité des stomates a varié de 33,88 à 50,32 stomate/ $\mathrm{mm}^{2}$. La plus grande valeur est observée chez la variété Pisifera et celle la plus faible chez le matériel Tenera prepépinière. Pour la longueur des stomates, il existe aussi une différence significative au seuil de $5 \%$ avec $P<0,0001$ entre les matériels sur les deux faces de feuille. Les valeurs de la longueur des stomates sur la face supérieure des feuilles ont varié de 34,46 à $55,16 \mu \mathrm{m}$. La variété Dura a des stomates ayant une longueur importante $(55,16$ um et le matériel Tenera prepépinière a les plus courts stomates $(34,46 \mu \mathrm{m})$. Sur la face inférieure des feuilles, les longueurs des stomates ont varié de 26,28 à $51,98 \mu \mathrm{m}$. La plus grande longueur est notée chez la variété Dura. Par contre la plus faible longueur est enregistrée chez le matériel Tenera prepépinière. S'agissant de La largeur moyenne des stomates, il existe une différence significative au seuil de $5 \%$ avec $P<0,0001$ entre les matériels sur les faces de feuille. Sur la face supérieure des feuilles la valeur de la largeur des stomates a varié de 18,57 à 39,41 $\mu \mathrm{m}$. La plus grande valeur de la largeur est observée chez la variété Pisifera et la plus faible valeur est notée chez le matériel prepépinière.

Tableau1 : Analyse statistique de la densité, longueur et largeur des stomates sur la face supérieure des feuilles du palmier à huile

\begin{tabular}{|l|l|l|l|l|l|l|l|}
\hline & \multicolumn{7}{|c|}{ Face supérieure des feuilles } \\
\cline { 2 - 8 } & F de Fisher & $\mathrm{P}$ associé & dura & pisifera & tenera & tenera $\mathrm{P}$ & tenera $\mathrm{PP}$ \\
\hline Densité & 20,58 & $<0,0001^{*}$ & $4,11^{\mathrm{b}}$ & $4,77^{\mathrm{b}}$ & $4,33^{\mathrm{b}}$ & $9,21^{\mathrm{a}}$ & $8,21^{\mathrm{a}}$ \\
\hline Longueur & 56,16 & $<0,0001^{*}$ & $55,16^{\mathrm{a}}$ & $54,88^{\mathrm{a}}$ & $53,05^{\mathrm{a}}$ & $39,91^{\mathrm{b}}$ & $34,46^{\mathrm{c}}$ \\
\hline largeur & 81,97 & $<0,0001^{*}$ & $39,38^{\mathrm{a}}$ & $39,41^{\mathrm{a}}$ & $38,63^{\mathrm{a}}$ & $28,13^{\mathrm{b}}$ & $18,57^{\mathrm{c}}$ \\
\hline
\end{tabular}

* différence significative au seuil de $5 \%$ avec la probabilité $P$ associée .Les moyennes portant en exposant la même lettre pour un même paramètre ne sont pas significativement différentes au seuil de risque de $5 \%$ selon le test de Fisher.

Tableau 2 : Analyse statistique de la densité, longueur et largeur des stomates sur la face inferieure des feuilles du palmier à huile

\begin{tabular}{|l|l|l|l|l|l|l|l|}
\hline & \multicolumn{7}{|c|}{ Face inférieure des feuilles } \\
\cline { 2 - 8 } & $\mathrm{F}$ de Fisher & $\mathrm{P}$ associé & dura & pisifera & tenera & tenera $\mathrm{p}$ & tenera $\mathrm{pP}$ \\
\hline Densité & 22,16 & $<0,0001^{*}$ & $43,54^{\mathrm{b}}$ & $50,32^{\mathrm{a}}$ & $42,88^{\mathrm{b}}$ & $35,72^{\mathrm{c}}$ & $33,88^{\mathrm{c}}$ \\
\hline Longueur & 37,20 & $<0,0001^{*}$ & $51,98^{\mathrm{a}}$ & $50,87^{\mathrm{a}}$ & $47,95^{\mathrm{a}}$ & $36,70^{\mathrm{b}}$ & $26,28^{\mathrm{c}}$ \\
\hline largeur & 56,12 & $<0,0001^{*}$ & $37,27^{\mathrm{a}}$ & $36,81^{\mathrm{a}}$ & $34,85^{\mathrm{a}}$ & $26,67^{\mathrm{b}}$ & $18,35^{\mathrm{c}}$ \\
\hline
\end{tabular}

* différence significative au seuil de $5 \%$ avec la probabilité $\mathrm{P}$ associé. Les moyennes portant en exposant la même lettre pour un même paramètre ne sont pas significativement différentes au seuil de risque de $5 \%$ selon le test de Fisher.

\section{DISCUSSION}

La densité stomatique est le nombre de stomates par unité de surface, elle varie en fonction des variétés, de l'âge des plants et des faces des feuilles. La forte valeur de la densité stomatique notée chez pisifera se rapproche de celle obtenue dans des essais similaires faits par Özeker et Misirli(2001) qui ont montré que chez Pistacia spp mâle, on a 353,38 stomates par $\mathrm{mm}^{2}$ contre 308,63 stomates par $\mathrm{mm}^{2}$ chez la femelle. La variété pisifera est utilisée comme parent mâle dans les schémas de sélection et production de semence du palmier à huile, il fait preuve d'un développement végétatif plus vigoureux, alors que dura et tenera ont des caractères végétatifs identiques (Beinaert et Vanderweyen, 1941). Ces auteurs considèrent que cette différence n'est pas due à la non production de régimes par le pisifera, puisqu'ils constatent une supériorité du pisifera bien avant l'entrée en production. Entre 18-20 mois de plantation, on observe déjà un développement végétatif plus important pour le pisifera. Ces mêmes auteurs concluent que cette différence serait d'ordre génétique et s'accentuerait avec l'âge par suite de l'avortement des régimes. II découle donc de toutes ces observations, qu'il y a une nette différence entre les matériels adultes et les matériels jeunes pour la densité stomatique. Les matériels jeunes 
ont moins de stomates que les matériels adultes sur la face inférieure des feuilles. Cette situation peut s'expliquer par le fait que les jeunes feuilles n'ont pas atteint leur croissance optimale permettant à certains stomates restés silencieux d'être fonctionnels. II est également à noter que les matériels jeunes ont une densité stomatique élevée sur la face supérieure des feuilles. D'une manière générale, les résultats obtenus sur la face inferieure des feuilles se rapprochent de ceux obtenus chez neuf variétés de blé dur dont les valeurs varient de 49,03 et 58 stomates par $\mathrm{mm}^{2}$ (Erchidi et al., 2000). Chez Ficus benjamina(L). La densité stomatique varie entre 247 et 310 stomates par $\mathrm{mm}^{2}$ sur la face inférieure des feuilles (Mounir et Raoult, 2000). Une étude similaire effectuée chez le macabo Xanthosoma sp par Njofang (1993) montre que la densité stomatique varie entre 80 et 109 stomates par $\mathrm{mm}^{2}$. Par contre chez les variétés de gombo, la densité stomatique varie de 21052,63 à 31926,83 stomates par $\mathrm{mm}^{2}$ sur la face inferieure et de 10701,75 à 15964,91 stomates par mm² sur la face supérieure des feuilles (Nana et al., 2010). Sur la face supérieure des feuilles, les valeurs obtenues sont très faibles mais les études ont montré que chez certaines espèces telles que le pommier, il n'existe pas des stomates sur la face supérieure des feuilles chez les variétés adultes (Heller, 1984). En dehors de ces constats, on note également que les densités moyennes des stomates sont plus importantes sur les faces inférieures que sur les faces supérieures des feuilles chez toutes les variétés. Ces résultats ont été aussi confirmés par les travaux de Özeker et Misirli(2001) dont les valeurs sont respectivement 353,78 stomates sur face abaxialeet 141,87 stigmates sur la face adaxiale. Chacune des variétés a donc une densité stomatique qui diffère suivant les faces de la feuille. Ces résultats sont semblables à ceux de Mbaye et al. (2001) et Njofang(1993). Cette différence peut être justifiée par la forte évapotranspiration sur la face inferieure que sur la face supérieure des feuilles, montrées par Heller (1984). Le nombre important de stomates par unité de surface $\left(\mathrm{mm}^{2)}\right.$ sur la face inférieure soit important constitue un atout pour la comparaison des variétés. Pour tous ces résultats, pour mieux comprendre nos données expérimentales, il faudrait tenir compte des conditions expérimentales. En effet, l'âge des feuilles et les conditions du milieu doivent être maîtrisés. Schoch (1972) et d'autres auteurs ont montré que la densité stomatique d'un végétal était fonction du rayonnement global. Cette densité stomatique peut être réduite de façon considérable par un ombrage croissant et varié, ainsi que dans les rapports de 1 à 10 , après l'arrêt total de la croissance foliaire. La densité stomatique n'est pas fonction uniquement du patrimoine génétique, mais dépend aussi pour une grande part des conditions de culture et d'éclairement (Dublin, 1974). II est par conséquent impératif, lors d'une étude comparative de ces caractères chez des individus, que ceux-ci soient soumis aux mêmes conditions d'éclairement et soient d'âge comparable. La longueur moyenne des stomates varie aussi suivant la variété, les faces des feuilles et l'âge des plants. Les résultats obtenus sont à peu prés comparables à ceux trouvés chez le blé dur dont les longueurs varient entre 61,51 et $47,79 \mu \mathrm{m}$ sur la face inférieure des feuilles (Erchidi et al., 2000). Chez le chou de Bruxelles, les mêmes essais réalisés par Dore (1986) ont montré que la longueur moyenne des cellules de garde des stomates chez les diploïdes est d'environ 20 $\mu \mathrm{m}$ et chez les polyploïdes, elle est supérieure à $24 \mu \mathrm{m}$. La longueur des stomates varie de 27,11 à $36,28 \mu \mathrm{m}$ chez Pistacia spp sur la face supérieure alors qu'elle varie de 25,71 à $34,45 \mu \mathrm{m}$ sur la face inférieure des feuilles (Özeker et Mirsili, 2001). Chez le macabo la longueur des stomates varie entre 27,3 et $30,3 \mu \mathrm{m}$ sur la face inférieure des feuilles Njofang,(1993). Ces résultats confirment les nôtres, car on constate aussi que la taille des stomates sur la face supérieure des feuilles est plus importante. Cette dimension peut être due au nombre réduit des stomates sur la face supérieure des feuilles, plus ce nombre est réduit plus les stomates se développent mieux. Le résultat révèle également que sur les deux faces des feuilles, les variétés tenera pépinière et tenera prépépinière ont des stomates moins longs. Cette réduction de taille chez ces matériels peut être due à leur état juvénile (croissance non parfaite). II y a donc une différence nette entre les matériels adultes et les matériels jeunes, entre les faces de feuilles.

S'agissant de la largeur des stomates, elle varie également en fonction de variétés, de l'âge des plants et les faces des feuilles. II ressort de l'analyse de ces résultats que les stomates de matériels adultes et les matériels jeunes ne sont pas les même du point de vue largeur. II en résulte également que les stomates de la face supérieure des feuilles sont plus larges que ceux qui existent sur la face inférieure des feuilles. La largeur des stomates varie de 17,14 à 20,72 $\mu \mathrm{m}$ chez Pistacia spp sur la face supérieure des feuilles, alors que sur la face inférieure des feuilles elle va de 15,27 à 20,59 um Özeker et Mirsili(2001).Cette différence entre la face supérieure et la face inférieure s'expliquerait par la densité des stomates sur les faces des feuilles. Par des études similaires, la largeur moyenne des stomates chez le macabo varie entre 19,1 et $26,7 \mu \mathrm{m}$ sur la face 
supérieure et, elle varie entre 14,8 et $21,1 \mu \mathrm{m}$ sur la face inférieure des feuilles Njofang(1993). Ces différents résultats se rapprochent des nôtres. Pour ce paramètre on remarque aussi de différences entre les faces supérieure et inferieure des feuilles. Les résultats ainsi obtenus, peuvent nous permettre de réaliser avec attention l'étude de la variation des paramètres d'évaluation du niveau de ploïdie des différentes variétés

\section{CONCLUSION}

Cette étude a permis d'évaluer les paramètres caractéristiques des stomates. Les résultats présentés confirment le bien fondé et l'efficacité des stomates dans l'évaluation du niveau de ploïdie chez Elaeis guineensis Jacq. actuel. Sur la face inferieure, la densité stomatique est plus élevée chez les matériels adultes que chez les matériels jeunes. Par contre, sur la face supérieure des feuilles, les matériels jeunes ont plus de stomates. La face inférieure apparaît comme la plus indiquée pour du palmier à huile. Quel que soit le paramètre étudié, nous signalons que la face supérieure présente plus de variation que la face inférieure des feuilles. Ainsi, la face inférieure des feuilles semble être la plus stable et la mieux indiquée pour évaluer le niveau de ploïdie de la plante Dore (1986).Des différences entre les cultivars de macabo pour les paramètres précités ont été montrées par Dang (1996) et Njofang (1993).

déterminer le niveau de ploïdie. Les stomates sur la face supérieure des feuilles sont plus longs et larges que ceux trouvés sur la face inferieure des feuilles. Cependant, les stomates sont plus longs que ceux des matériels jeunes. Les résultats obtenus peuvent s'appliquer dans la recherche des variétés vigoureuses et productrices en se basant sur la polyploïdisation. Ainsi, ce critère mis en évidence peut être judicieusement utilisé par les sélectionneurs pour améliorer le palmier à huile.

\section{REMERCIEMENTS}

Nous tenons à remercier l'Université de Yaoundé I et l'Université Ouagal Pr Joseph KI-ZERBO qui ont soutenu matériellement, financièrement et moralement ce travail.

\section{BIBLIOGRAPHIE}

Auge R, Beauchesne G, Boccon-Gibod J, Decourtye L, Digat B, Jalouzot R, Minier R, Morand JC, Reynoird JP,Strullu G, Vidalie H, 1989. La culture in vitro et ses applications horticoles. Lavoisier. Paris. $225 \mathrm{p}$.

Beinaert $A$, Vanderweyen $R, 1941$. Contribution à l'étude génétique et biométrique des variétés d'Elaeis guineensis Jacq. Publ de l'INEAC, sér. sci. 27 : 1-101.

Dang MJ, 1996.Comparaison des paramètres biométriques des stomates chez le macabo (Xanthosoma sp.). Mémoire de Maîtrise en sciences de la vie, Univ. Yaoundé I. $43 \mathrm{p}$.

Demol J, 2002. Amélioration des plantes, application aux principales espèces cultivées en régions tropicales. Presses agronomiques. Gembloux. $560 \mathrm{p}$.

Dore C, 1986. Évaluation du niveau de ploïdie des plantes d'une population de choux de Bruxelles (Brassicaoleracea L. Gemmifera) d'origine pollinique. Agro. 6 (9) : 797-801

Dublin P, 1974. Les haploïdes de Theobroma cacao L. diplöidisation et obtention d'individus homozygotes. Café Cacao Thé. 18 (2) : 83-96.

Dubois J, 1989. Biotechnologie et amélioration des plantes. In Plantes vivrières tropicales.
AupelfUref (ed.). Paris John Libbey Eurotext. 1925.

Erchidi AE, Benbella M, Talouizte A, 2000. Relation entre certains paramètres contrôlant les pertes en eau et le rendement grain chez neuf variétés de blé dur soumis au stress hydrique Cah.-Opt. Mid. : sér $A$. sém, 40 : 279-282.

Heller R, 1984. Abrégé de physiologie végétale.1. Nutrition. (3eed) Masson. Paris 246p.

Koffi AN, Barima SSY, Angaman MD, Dongui KB, 2014. Les caractéristiques des stomates des feuilles de Ficus benjamina L. comme bioindicateurs potentiels de la qualité de l'air dans la ville d'Abidjan (Côte d'Ivoire). J. Appl. Biosci. 78 : 6675-6684.

Mbaye.MS, Noba K, Sarr RS, Sambou JM, Amadou Tidiane BA, 2001. Élément de précision sur la systématique d'espèces Adventices du genre Corchorus L. (Tiliacée) au Sénégal. Af. J. of Sci and Tech. 2(1) : 51-64.

Mounir D, Raoult L, 2000. Modélisation de la résistance stomatique en fonction des caractéristiques morphologiques et anatomiques des stomates, du rayonnement et du potentiel hydrique. Sci et chan planét/séch 11(1) : 29-36. 
Nana R, Tamini Z, Some PP, 2010. Étude morphologique comparative de cinq variétés de gombo (Abelmoschus esculentus (L.) MOENCH) soumises à un stress hydrique. J. Sci., 10(3) : $28-38$.

Njofang C, 1993. Recherche d'un critère d'évaluation du niveau de ploïdie chez Xanthosoma sp.: Densité et dimensions des stomates. Mémoire de Maîtrise en Sciences de la vie. Univ. Yaoundé I. $47 \mathrm{p}$.
Özeker E, Misirli A, 2001. Research on leaf properties and stomata distribution of some Pistacia spp Cah Opt Med. 11(56) 237-241.

Schoch PG, 1972. Variation de la densité stomatique de Capsicum annuum $\mathrm{L}$. en fonction $\mathrm{du}$ rayonnement global. C.r. Acad. Sci., 274. 24962498.

Scienza A, Boselit M, 1981. Fréquence et caractéristiques biométriques des stomates de certains porte-greffes de vigne. Vitis 20, 281292. 